\title{
Estudo Comparativo entre a Síndrome Antifosfolípide Primária e a Secundária - Características Clínico-laboratoriais em 149 Pacientes
}

\author{
Comparative Study Between Primary and Secondary \\ Antiphospholipid Syndrome - Clinic and Laboratorial \\ Characteristics of 149 Patients
}

\author{
Caio Robledo D’Angioli Costa Quaio ${ }^{(1)}$, Paulo Eduardo Daruge Grando(1) ${ }^{(1)}$ Jozélio Freire de Carvalho $^{(2)}$
}

\section{RESUMO}

Objetivos: O presente estudo tem como objetivo analisar as características clínicas, laboratoriais e imunológicas dos pacientes com síndrome antifosfolípide (SAF) e comparar indivíduos portadores da síndrome primária com portadores da secundária. Pacientes e Métodos: Foi analisado o banco de dados de 149 pacientes com SAF do Serviço de Reumatologia do HC-FMUSP que satisfaziam os critérios de classificação para SAF. Resultados: A amostra consistiu de 140 (94\%) mulheres e 9 (6\%) homens, com média de idade de 39,7 $\pm 11,6$ anos. A SAF primária perfez o total de $50(33,8 \%)$ pacientes. As tromboses arteriais foram mais significativamente freqüentes no grupo de $\mathrm{SAF}$ primária em relação à secundária $(56 \%$ versus $35,7 \%$, $\mathrm{p}=0,02)$, mais especificamente a presença da síndrome de Sneddon ( $8 \%$ versus $0, \mathrm{p}=0,012)$ e isquemia de extremidades ( $18 \%$ versus $5 \%$, $\mathrm{p}=0,017)$. Com exceção de catarata, que foi mais freqüente no grupo associado ao LES ( $10 \%$ versus $0, \mathrm{p}=0,017)$, não se observou diferenças significativas em relação às outras comorbidades. Em relação aos auto-anticorpos, o grupo de SAF secundária apresentou significativamente maior prevalência de $\mathrm{FAN}(99 \%$ versus $60 \%, \mathrm{p}<$ $0,0001)$, anti-ENA $(45,9 \%$ versus $22 \%, \mathrm{p}=0,007)$ e anti-Ro/SS-A $(43 \%$ versus $8 \%, \mathrm{p}<0,0001)$. Com exceção da anticardiolipina IgG que foi mais freqüentemente observada no grupo de SAF secundária $(84,7 \%$ versus $60 \%, \mathrm{p}=0,0018)$, os outros anticorpos antifosfolípides (anticardiolipina $\operatorname{IgM}$ e anticoagulante lúpico) não diferiram entre os grupos. Conclusões: O presente estudo identificou população de SAF primária que apresenta maior freqüência de fenômenos trombóticos arteriais, mais especificamente a síndrome de Sneddon e isquemia de extremidades, em relação aos pacientes com SAF secundária. Reforçou, também, o papel de auto-anticorpos na identificação de subgrupos de SAF associada ao LES.

Palavras-chave: síndrome antifosfolípide, epidemiologia, lúpus eritematoso sistêmico, trombofilia, anticardiolipina.

\begin{abstract}
Objectives: The aim of this study was to analyze the prevalence and characteristics of the main clinical, immunologic and laboratorial features of antiphospholipid syndrome (APS), and to perform a comparison between primary and secondary forms of APS. Patients and Methods: A data base of 149 patients from HCFMUSP who met the preliminary criteria for the classification of APS was analyzed. Results: The pattern consisted of $140(94 \%)$ female and $9(6 \%)$ male patients with a mean age of $39.7 \pm 11$.6years. Primary APS was present in 50 (33.8\%) of the patients. Arterial thrombosis were more found in primary APS compared to secondary APS (56\% vs. 35.7\%, p = 0.02), more specifically the presence of Sneddon's syndrome (56\% vs. $35,7 \%, p=0.02)$ and limbs ischaemia (18\%vs. 5\%, $p=0.017)$ were more prevalent in the first group. Except cataract that was more frequently observed in secondary APS group (10\% vs. 0 , $p=0.017$ ), no other significant difference was found regarding comorbidities. In relation to autoantibodies, the secondary APS patients had a more significant prevalence of ANA (99\% vs. 60\%, $p<0.0001)$, anti-ENA (45.9\% vs. 22\%, p = 0.007) e anti-Ro/ SS-A (43\% vs. 8\%, p 0.0001). Antiphospholipid antibodies (IgM anticardiolipin and lupus anticoagulant) did not differ between the groups, except IgG anticardiolipin that was more found in secondary APS group (84.7\% vs. 60\%, p =0.0018), Conclusions: the present study showed that primary APS had more arterial thrombotic events, more specifically Sneddon's syndrome and limbs ischemia, than secondary APS. It was also reinforced the role of autoantibodies to identify patients with APS associated to SLE.
\end{abstract}

Keywords: antiphospholipid syndrome, epidemiology, systemic lupus erythematosus, thrombophilia, anticardiolipin.

Recebido em 8/7/2008. Aprovado, após revisão, em 10/7/2008. Declaramos a inexistência de conflitos de interesse.

1. Médico colaborador do Hospital das Clínicas da Faculdade de Medicina da Universidade de São Paulo (HC-FMUSP).

2. Professor-colaborador da disciplina de Reumatologia da FMUSP e médico-assistente doutor, chefe do Ambulatório de Síndrome Antifosfolípide do Serviço de Reumatologia do HC-FMUSP.

Endereço para correspondência: Jozélio Freire de Carvalho, Disciplina de Reumatologia da Faculdade de Medicina da Universidade de São Paulo, Av. Dr. Arnaldo, 455, $3^{\circ}$ andar, sala 3190, Cerqueira César, 01246-903, São Paulo, SP, e-mail: jotafc@gmail.com 


\section{INTRODUÇÃO}

A síndrome antifosfolípide (SAF) refere-se à condição clínica caracterizada pela presença de tromboses vasculares de repetição e/ou quadros de abortamentos de repetição, sempre associada à presença de anticorpos antifosfolípides $(\mathrm{AAF})^{(1,2)}$.

A SAF pode ser primária quando aparece isoladamente, sem nenhuma outra condição auto-imune associada, ou vir associada a uma dessas condições, principalmente o lúpus eritematoso sistêmico (LES), sendo primeiramente descrita em pacientes com esta condição clínica ${ }^{(3)}$. Nessa circunstância, é dita SAF secundária ao LES. No universo de pacientes com LES, a prevalência de anticardiolipina pode variar de $12 \%$ a $30 \%(4,5)$ e do anticoagulante lúpico pode variar de $15 \%$ a $34 \%{ }^{(4,6)}$. A manifestação clínica da SAF ocorre em $50 \%$ a $70 \%$ dos pacientes com LES que apresentam os $\mathrm{AAF}^{(7,8)}$. A presença do anticorpo aumenta o risco de trombose em cerca de cinco vezes e a história prévia de trombose também aumenta o risco de um novo evento vascular trombótico ${ }^{(3)}$.

Estudos recentes agregaram importantes conhecimentos acerca das manifestações da SAF, com grande destaque à coorte européia ${ }^{(9)}$, com mil casos acompanhados, que trouxeram novos conhecimentos sobre esses subgrupos de pacientes.

Embora inúmeras dessas manifestações tenham sido descritas $^{(1,10-12)}$, suas reais prevalências ainda geram incertezas. Entre as manifestações, enumeram-se: isquemia de extremidades, abortos espontâneos, óbitos fetais, partos prematuros, tromboses venosas, valvopatias, síndrome de Sneddon, acometimento renal, amaurose fugaz, neurite óptica e osteonecrose.

Igualmente incertas são as reais diferenças entre as síndromes primária e secundária da SAF. No Brasil, há carência de estudos epidemiológicos a respeito do presente tópico ${ }^{(13)}$. Sabe-se, ainda, que existem grandes variações étnicas na expressão clínica da $\operatorname{SAF}^{(1,9-12,14,15)}$.

O presente estudo tem como objetivo analisar as características demográficas, clínicas, laboratoriais e imunológicas de uma coorte de pacientes com SAF e comparar características distintivas da doença entre as síndromes primária e secundária de determinada população brasileira.

\section{PACIENTES E MÉTODOS}

\section{SELEÇÃO DOS PACIENTES}

O presente estudo foi realizado no período de 1995 a 2005 , por meio da análise de 1.451 prontuários disponíveis no sistema eletrônico (ProntMed - Prontuário Eletrônico 3.0, ProntMed, São Paulo, SP, Brasil) e prontuários convencionais utilizados pelo Serviço de Reumatologia do Hospital das Clínicas da Faculdade de Medicina da Universidade de São Paulo (FMUSP). Desse universo, foram excluídos os casos que não satisfaziam os critérios de classificação para $S F^{(16)}$, obtendo-se, ao final, 149 pacientes que foram incluídos.

Foram colhidos dados demográficos, clínicos e laboratoriais dos pacientes dos 149 casos. As comorbidades e os fatores de risco cardiovasculares associados também foram analisados, como a presença de hipertensão arterial, diabetes melito, tabagismo, uso de anticoncepcional oral, terapia de reposição hormonal, dislipidemia e etilismo. Os medicamentos utilizados pelos pacientes na última consulta (incluindo corticoterápicos, agentes imunossupressores e anticoagulantes) foram anotados. Os pacientes foram posteriormente divididos em dois grupos: aqueles com SAF primária, que incluía os casos que preenchiam critérios para os diagnósticos anteriormente relacionados e sem indícios de qualquer outra doença reumatológica auto-imune (por exemplo, LES, esclerose sistêmica, síndrome de Sjögren, vasculites sistêmicas, miosites e artrite reumatóide) e SAF secundária, quando associada ao LES, segundo os critérios do American College of Reumatology (ACR) ${ }^{(17)}$ ou LES-símile quando menos do que quatro dos critérios de classificação de LES estavam presentes. Registros da ocorrência de tromboses e dos seus respectivos locais de acometimento, juntamente com a história de complicações gestacionais foram coletados. Os casos de abortamentos provocados foram excluídos da casuística.

\section{AVALIAÇÃO LABORATORIAL}

Os anticorpos antinucleares (ANA) foram detectados pela técnica de imunofluorescência indireta utilizando imprint de fígado de camundongo e células HEp2 como substratos. Foram considerados resultados positivos aqueles acima de $1: 40$.

Os anticorpos dirigidos contra antígenos solúveis (antiUIRNP, anti-Ro/SS-A e anti-La/SS-B) em solução salina foram detectados por contra-imunoeletroforese em gel de agarose a 1,5\% em tampão barbital, $\mathrm{pH} 8,2^{(18)}$. Soros referências comerciais com especificidades antigênicas conhecidas foram utilizados para caracterizar os anticorpos precipitantes. Extratos de baço de cachorro e de timo de 
coelho (Sigma Chem Co. St Louis, EUA) foram utilizados como fonte de antígenos ${ }^{(19)}$.

Os anticorpos anticardiolipina (aCL) foram detectados por ELISA ${ }^{(20)}$. Resumidamente, orifícios de placas de poliestireno foram sensibilizados com a cardiolipina (Sigma Chem. Co., EUA), na concentração de $50 \mathrm{ug} / \mathrm{ml} \mathrm{em}$ etanol gelado ( $50 \mathrm{ul} /$ orifício), por 16 horas a $4{ }^{\circ} \mathrm{C}$. Após lavagem, a placa foi bloqueada por 2 horas com soro fetal bovino inativado $\left(56{ }^{\circ} \mathrm{C}\right.$, por 30 minutos) a $30 \%$ em PBS. Os orifícios foram seqüencialmente incubados, em duplicata, com os soros diluídos 1:50 e IgG de cabra anti-IgG ou anti-IgM humanas, marcadas com peroxidase (Sigma Chem. Co., EUA). Os resultados foram expressos em unidades GPL e MPL, determinadas por meio de curva construída a partir dos valores de densidade óptica obtidos de amostras de soros referência definidos internacionalmente. Foram considerados positivos para este estudo aqueles soros $>10 \mathrm{U}$. Para o diagnóstico da síndrome aqueles acima de $20 \mathrm{U}$, segundo os critérios de Sapporo, ${ }^{(16)}$.

O anticoagulante lúpico (LAC) foi realizado por meio de testes hematológicos funcionais inicialmente pelo teste de rastreamento com o tempo de tromboplastina parcial ativada (TTPa) e, em seguida, o teste do veneno da víbora de Russel. Em caso de alteração, foi realizada a prova de mistura de $50 \%$ de plasma normal com $50 \%$ do plasmateste, caso houvesse correção do TTPa foi diagnosticada deficiência de fator de coagulação. Caso contrário, se não houvesse correção e esta fosse obtida com a mistura com plasma rico em fosfolípides (plaquetas) estava diagnosticado o LAC.

\section{ANÁLISE ESTATÍSTICA}

Os resultados foram apresentados em médias, desviospadrão e porcentagens, sendo fornecidas as variações mínima e máxima e, em alguns parâmetros, a mediana. Os dados foram comparados pelo teste $t$ de Student para variáveis paramétricas para avaliar a diferença entre os grupos SAF primário e secundário. As diferenças de porcentagens foram comparadas pelo teste exato de Fisher. A significância estatística foi considerada quando o p-valor fosse menor que 0,05 .

\section{RESULTADOS}

Em relação a toda a população estudada, a média de idade foi de $39,7 \pm 11,6$ (variando de 2 a 72 anos), sendo o sexo feminino (94\%) o mais prevalente. A cor branca
$(60,4 \%)$ foi a predominante e a média de duração da doença SAF foi de $7,7 \pm 5,5$ anos. Pacientes com trombose venosa perfaziam $48,3 \%$ e aqueles com fenômenos arteriais compreenderam $42,3 \%$.

Cinqüenta $(33,8 \%)$ pacientes eram portadores da síndrome primária, e os $98(66,2 \%)$ restantes perfaziam o grupo associado à LES.

As idades médias, a freqüência de sexo feminino e de cor branca, bem como a duração da doença não diferiram significativamente entre ambos os grupos de SAF primária e secundária (Tabela 1 ).

TABELA 1

Características DEMOGRÁFICAS E CLÍNICAS ENTRE PACIENTES COM SÍNDROME ANTIFOSFOLÍPIDE PRIMÁRIA E SECUNDÁRIA

\begin{tabular}{|c|c|c|c|}
\hline & $\begin{array}{l}\text { SAF primária } \\
\mathrm{n}=50\end{array}$ & $\begin{array}{l}\text { SAF secundária } \\
\mathrm{n}=98\end{array}$ & $\mathbf{p}$ \\
\hline Média de idade (anos) & $40,6 \pm 11,7$ & $39,4 \pm 11,5$ & 0,54 \\
\hline Sexo feminino $\mathrm{n}(\%)$ & $47(94)$ & $92(93,9)$ & 1,00 \\
\hline Cor branca n (\%) & $29(58)$ & $61(62)$ & 0,72 \\
\hline Duração da SAF (anos) & $7,2 \pm 5,4$ & $8,0 \pm 5,5$ & 0,38 \\
\hline Trombose venosa $\mathrm{n}(\%)$ & $18(36)$ & $45(46)$ & 0,29 \\
\hline Trombose arterial $\mathrm{n}(\%)$ & $28(56)$ & $35(35,7)$ & 0,02 \\
\hline $\begin{array}{l}\text { Trombose de pequenos vasos } \\
\text { observada na histologia } n(\%)\end{array}$ & $4(8)$ & $4(4)$ & 0,44 \\
\hline Infarto agudo do miocárdio $\mathrm{n}(\%)$ & $1(2)$ & $1(1)$ & 1,00 \\
\hline $\begin{array}{l}\text { Acidente vascular cerebral } \\
\text { isquêmico } \mathrm{n}(\%)\end{array}$ & $16(32)$ & $26(26,5)$ & 0,56 \\
\hline Tromboembolismo pulmonar $\mathrm{n}(\%)$ & $5(10)$ & $12(12)$ & 0,79 \\
\hline Isquemia de extremidade $n(\%)$ & $9(18)$ & $5(5)$ & 0,017 \\
\hline Abortos espontâneos $n(\%)$ & $12(24)$ & $24(24,5)$ & 1,00 \\
\hline Óbitos fetais $\mathrm{n}(\%)$ & $10(20)$ & $13(13)$ & 0,34 \\
\hline Partos prematuros $\mathrm{n}(\%)$ & $6(12)$ & $12(12)$ & 1,00 \\
\hline Valvopatias $\mathrm{n}(\%)$ & $2(4)$ & $3(3)$ & 1,00 \\
\hline Síndrome de Sneddon $\mathrm{n}(\%)$ & $4(8)$ & 0 & 0,012 \\
\hline $\begin{array}{l}\text { Renal (microangiopatia trombótica) } \\
\mathrm{n}(\%)\end{array}$ & $3(6)$ & $1(1)$ & 0,11 \\
\hline Amaurose fugaz $\mathrm{n}(\%)$ & 0 & $2(2)$ & 0,55 \\
\hline Neurite óptica n(\%) & $2(4)$ & 0 & 0,11 \\
\hline Osteonecrose $\mathrm{n}(\%)$ & 0 & $3(3)$ & 0,55 \\
\hline
\end{tabular}

$\mathrm{SAF}=$ síndrome antifosfolípide.

Em relação às manifestações clínicas, a freqüência de trombose arterial seja em vasos periféricos ou em vasos do sistema nervoso central foi significativamente maior no grupo SAF primária ( $56 \%$ versus $35,7 \%, \mathrm{p}=0,02)$, sendo 
os fenômenos clínicos arteriais associados à isquemia de extremidades ( $18 \%$ versus $5 \%, \mathrm{p}=0,017)$ e à presença da síndrome de Sneddon ( $8 \%$ versus $0, \mathrm{p}=0,012)$ comparado ao grupo de SAF secundária. A freqüência das outras manifestações vasculares da doença, como trombose venosa ou de pequenos vasos, infarto agudo do miocárdio (IAM), acidente vascular cerebral (AVC) isquêmico, tromboembolismo pulmonar (TEP) foram similares nos dois grupos. Em relação às manifestações obstétricas (abortos espontâneos, partos prematuros e óbitos fetais) também não foram observadas diferenças significativas comparando ambos os grupos. Aa alterações do sistema oftalmológico (neurite óptica e amaurose fugaz), do sistema renal, do coração (valvopatias) e osteonecrose foram também semelhantes nos dois grupos de pacientes (Tabela 1 ).

Em relação às comorbidades mais comuns, comparando-se o grupo de SAF primária e SAF secundária, excetuando-se pela presença mais freqüente de catarata no grupo associado ao LES (0 versus $10 \%, \mathrm{p}=0,017$ ), não houve diferença significativa em relação à presença de hipertensão arterial sistêmica, tabagismo, hipotireoidismo, depressão, osteopenia/osteoporose e história de tuberculose (Tabela 2).

TABELA 2

COMORBIDADES ENTRE PACIENTES COM SÍNDROME ANTIFOSFOLÍPIDE PRIMÁRIA E SECUNDÁRIA

\begin{tabular}{lccc}
\hline & $\begin{array}{c}\text { SAF primária } \\
\mathrm{n}=50\end{array}$ & $\begin{array}{c}\text { SAF secundária } \\
\mathrm{n}=98\end{array}$ & $\mathrm{p}$ \\
\hline Hipertensão arterial sistêmica $\mathrm{n}(\%)$ & $11(22)$ & $26(26,5)$ & 0,69 \\
Diabetes melito n (\%) & $3(6)$ & $4(4)$ & 0,69 \\
Tabagismo n (\%) & $5(10)$ & $8(8)$ & 0,76 \\
Hipotireoidismo n (\%) & $4(8)$ & $8(8)$ & 1,00 \\
Depressão n (\%) & $4(8)$ & $7(7)$ & 1,00 \\
Osteopenia/osteoporose n (\%) & $1(2)$ & $9(9)$ & 0,17 \\
Fibromialgia n (\%) & $2(4)$ & $6(6)$ & 0,72 \\
História de tuberculose $\mathrm{n}(\%)$ & 0 & $4(4)$ & 0,30 \\
Catarata n (\%) & 0 & $10(10)$ & 0,017 \\
\hline
\end{tabular}

Considerando-se a presença de auto-anticorpos, a maioria destes foi mais freqüentemente encontrada naqueles pacientes com SAF associada ao LES. Desse modo, a presença de fator antinúcleo (60\% versus $99 \%$, $\mathrm{p}<0,0001)$, anti-ENA total ( $22 \%$ versus $45,9 \%, \mathrm{p}=0,007)$ e anti-Ro/SS-A ( $8 \%$ versus $43 \%, \mathrm{p}<0,0001)$ foram todos estatisticamente diferentes, comparando-se os dois grupos. Entretanto, a freqüência do anticorpo anti-La/SS-B apresentou similaridade, comparando-se os grupos (0 versus $8 \%, \mathrm{p}=0,052)$ (Tabela 3$)$.

Ao analisar os anticorpos relacionados à SAF, percebeu-se que não houve distinção significativa entre a presença dos anticorpos anticardiolipina do subtipo IgM e anticoagulante lúpico. Por outro lado, os anticorpos anticardiolipina $\mathrm{IgG}$ foram mais freqüentes no subgrupo de pacientes com SAF secundária $(84,7 \%$ versus $60 \%$, $\mathrm{p}=0,0018)$ em relação à SAF primária. Entretanto, ao se analisar a média dos anticorpos anticardiolipina, tanto IgG $(42,4 \pm 0,7$ versus $35,2 \pm 43,3 \mathrm{GPL}, \mathrm{p}=0,70)$ quanto IgM $(20,5 \pm 35,1$ versus $24 \pm 32,2 \mathrm{MPL}, \mathrm{p}=0,55)$, não foram observadas diferenças significativas nas duas populações de pacientes (Tabela 3 ).

TABELA 3

Perfil de Auto-Anticorpos entre pacientes COM SÍNDROME ANTIFOSFOLÍPIDE PRIMÁRIA E SECUNDÁRIA

\begin{tabular}{lccc}
\hline & $\begin{array}{c}\text { SAF primária } \\
\mathbf{n}=\mathbf{5 0}\end{array}$ & $\begin{array}{c}\text { SAF secundária } \\
\mathbf{n}=98\end{array}$ & $\mathbf{p}$ \\
\hline Fator antinúcleo (\%) & $30(60)$ & $97(99)$ & $<0,0001$ \\
Anti-ENA (\%) & $11(22)$ & $45(45,9)$ & 0,007 \\
Anti-Ro/SS-A (\%) & $4(8)$ & $43(43)$ & $<0,0001$ \\
Anti-La/SS-B (\%) & 0 & $8(8)$ & 0,052 \\
aCL IgG (\%) & $30(60)$ & $83(84,7)$ & 0,0018 \\
aCL IgM (\%) & $29(58)$ & $44(44,9)$ & 0,16 \\
aCL IgG, média, GPL & $42,4 \pm 0,7$ & $35,2 \pm 43,3$ & 0,70 \\
aCL IgM, média, MPL & $20,5 \pm 35,1$ & $24 \pm 32,2$ & 0,55 \\
Anticoagulante lúpico $\mathrm{n}(\%)$ & $22(44)$ & $37(37,8)$ & 0,48 \\
\hline
\end{tabular}

O estudo dos medicamentos utilizados pelos pacientes aqui estudados demonstrou freqüência significativa menor de uso de prednisona ( $14 \%$ versus $50 \%$, $\mathrm{p}<0,0001)$ e dose menor deste glicocorticóide $(3,2 \pm$ $10,6$ versus $8,4 \pm 13,3 \mathrm{mg} / \mathrm{dia}, \mathrm{p}=0,02)$ e, também, menor uso de azatioprina ( $2 \%$ versus $20 \%, \mathrm{p}=0,002)$ no grupo de SAF primária. Os dois grupos foram semelhantes em relação ao uso de warfarin, cloroquina, ácido acetilsalićlico, hipolipemiantes, uso de inibidores da enzima conversora de angiotensina ou bloqueadores do receptor de angiotensina II, diuréticos e uso de anticoncepcionais orais (Tabela 4). 
TABELA 4

DISTRIBUIÇÃO DE MEDICAMENTOS ENTRE PACIENTES COM SÍNDROME ANTIFOSFOLÍPIDE PRIMÁRIA E SECUNDÁRIA

\begin{tabular}{lccc}
\hline $\begin{array}{l}\text { Freqüência de uso } \\
\text { de medicamentos }\end{array}$ & $\begin{array}{c}\text { SAF primária } \\
\mathbf{n}=\mathbf{5 0}\end{array}$ & $\begin{array}{c}\text { SAF secundária } \\
\mathbf{n}=\mathbf{9 8}\end{array}$ & $\mathbf{p}$ \\
\hline Warfarin (\%) & $32(64)$ & $53(54,1)$ & 0,29 \\
Cloroquina (\%) & $23(46)$ & $61(62,1)$ & 0,08 \\
$\begin{array}{l}\text { Dose média de } \\
\text { prednisona (mg/dia) }\end{array}$ & $3,2 \pm 10,6$ & $8,4 \pm 13,3$ & 0,02 \\
Uso de prednisona (\%) & $7(14)$ & $49(50)$ & $<0,0001$ \\
Ácido acetilsalicílico (\%) & $4(8)$ & $12(12)$ & 0,58 \\
Azatioprina n (\%) & $1(2)$ & $20(20)$ & 0,002 \\
Hipolipemiante n (\%) & $8(9)$ & $14(14)$ & 0,81 \\
$\begin{array}{l}\text { Inibidor de enzima conversora } \\
\text { de angiotensina/Bloqueador do }\end{array}$ & $13(26)$ & $32(32,7)$ & 0,45 \\
receptor de angiotensina II (\%) & & & \\
$\begin{array}{l}\text { Diurético (\%) } \\
\text { Anticoncepcional oral } \mathrm{n}(\%)\end{array}$ & $11(22)$ & $18(18,4)$ & 0,66 \\
\hline
\end{tabular}

\section{DISCUSSÃO}

O presente estudo de uma coorte de pacientes com SAF conseguiu revelar algumas particularidades importantes quando se comparou pacientes com a síndrome isoladamente daqueles associados ao LES, em um corte transversal.

Nesse sentido, a presença de fenômenos trombóticos arteriais foi mais vista em pacientes com a síndrome primária, porquanto as manifestações arteriais significativamente mais encontradas foram aquelas da síndrome de Sneddon e da isquemia de extremidades. A síndrome de Sneddon está em concordância com a literatura, no sentido de que o sítio arterial mais encontrado na SAF seja o AVC. Essa síndrome caracteriza-se pela presença de AVC associado à livedo reticular.

Em um estudo dessa doença em um serviço terciário, como é o Hospital das Clínicas, é quase certa a presença de viés de seleção de casos mais graves e com maior complexidade, em razão da hierarquia do sistema de saúde. Dentro dessa perspectiva, nota-se, por exemplo, maior tendência de notificação, investigação e tratamento de pacientes que apresentaram eventos trombóticos arteriais em relação aos venosos. Isso pode ocorrer, em especial, nos casos de SAF primária. Portanto, essa variável deve ser levada em consideração nos grupos de pacientes acompanhados em centros universitários.

Não obstante terem sido descritos subtipos de SAF agregados a faixas etárias, sexo e $\operatorname{cor}^{(9)}$, não foram encontradas diferenças estatísticas entre os grupos etários e de cor aqui avaliados. Talvez, em vez de subgrupos, tenha-se, na realidade, um grande grupo étnico neste estudo que reflete a grande miscigenação da população brasileira.

Mesmo que subestimadas, as tromboses de origem venosa são ocorrências vasculares muito comuns. Nesse universo, a trombose venosa profunda (TVP) foi o evento mais freqüente. Essa incidência é maior que em uma coorte de população européia - 31,7\% -, que avaliou mil pacientes (dos quais $53,1 \%$ apresentaram critérios clínicos para SAF primária, $41,2 \%$ para LES ou LES-like) ${ }^{(9)}$ e muito menor que na população chinesa $-75 \%^{(15)}$. As tromboses arteriais estiveram presentes em $42,3 \%$ dos casos e apresentaram incidência bem maior quando comparadas com a população européia (13,1\%). O infarto agudo do miocárdio teve baixa incidência no presente estudo $(1,3 \%)$, sendo menor em comparação ao grupo europeu $(5,5 \%)$ e ao chinês $(4,2 \%)$.

Ao se comparar os grupos de pacientes com SAF primária com aqueles que apresentam SAF secundária, a presença de tromboses arteriais é estatisticamente maior na população de SAF primária. Esse resultado corrobora estudo epidemiológico brasileiro prévio ${ }^{(13)}$. No entanto, ao serem analisados eventos trombóticos específicos, só foram encontradas diferenças significativas em isquemia de extremidades e síndrome de Sneddon, ambas mais freqüentes nos doentes com SAF primária. Outros eventos trombóticos arteriais de grande importância, como o IAM e o acidente vascular cerebral isquêmico (AVCi), não apresentam diferenças significativas entre os grupos.

O presente estudo também ressalta que a presença de auto-anticorpos foi mais prevalente no grupo de pacientes com lúpus associado, refletindo talvez maior chance desses anticorpos nesse subgrupo específico, como já é de conhecimento.

Ao serem comparados os dados do presente estudo com outros estudos mundiais, essas diferenças populacionais encontradas não se devem somente ao regionalismo e às diferenças étnicas, mas tem-se de considerar, sempre, as diferenças logísticas dos diversos sistemas de saúde e as diferenças entre as dificuldades em realizar-se o diagnóstico, bem como manter o acompanhamento clínico eficaz em cada país.

Em conclusão, o presente estudo pôde identificar a prevalência de importantes características da SAF no Brasil, agregando conhecimentos acerca de sua apresentação e características demográficas da população acometida e aprofundando o estudo sobre as diferenças entre os grupos de SAF primária e secundária. A intenção do presente estudo é acompanhar esses pacientes e reunir maiores informações que permitam conclusões mais objetivas sobre o tema em estudos subseqüentes. 


\section{REFERÊNCIAS}

1. Alarcon-Segovia D, Deleze M, Oria CV, et al.: Antiphospholipid antibodies and the antiphospholipid syndrome in systemic lupus erythematosus. A prospective analysis of 500 consecutive patients. Medicine (Baltimore) 68: 353-65, 1989.

2. Hughes GRV, Harris EN, Gharavi AE: The anticardiolipin syndrome. J Rheumatol 13: 486-9, 1986.

3. Levine JS, Branch DW, Rauch J.: The antiphospholipid syndrome. N Engl J Med 346: 752-63, 2002.

4. Cervera R, Khamashta MA, Font J, et al.: Systemic lupus erythematosus: clinical and immunologic patterns of disease expression in a cohort of 1,000 patients. Medicine (Baltimore) 72: 113-24, 1993.

5. Merkel PA, Chang YC, Pierangeli SS, et al.: The prevalence and clinical associations of anticardiolipin antibodies in a large inception cohort of patients with connective tissue diseases. Am J Med 101: 576-83, 1996.

6. Love PE, Santoro SA: Antiphospholipid antibodies: anticardiolipin and the lupus anticoagulant in systemic lupus erythematosus (SLE) and in non-SLE disorders: prevalence and clinical significance. Ann Intern Med 112: 682-98, 1990.

7. Alarcón-Segovia D, Pérez-Vázquez ME, Villa AR, et al.: Preliminary classification criteria for the antiphospholipid syndrome within systemic lupus erythematosus. Semin Arthritis Rheum 21: 275-86, 1992.

8. Petri M. Epidemiology of the antiphospholipid antibody syndrome. J Autoimmun 15: 145-51, 2000.

9. Cervera R, Piette JC, Font J, et al. Euro-Phospholipid Project Group. Antiphospholipid syndrome: clinical and immunologic manifestations and patterns of disease expression in a cohort of 1,000 patients. Arthritis Rheum 46: 1019-27, 2002.

10. Cervera R, Font J, Lopez-Soto A, et al. Isotype distribution of anticardiolipin antibodies in systemic lupus erythematosus: prospective analysis of a series of 100 patients. Ann Rheum Dis 49: 109-13, 1990.
11. Font J, Lopez-Soto A, Cervera R, et al. The 'primary' antiphospholipid syndrome: antiphospholipid antibody pattern and clinical features of a series of 23 patients. Autoimmunity 9: 69-75, 1991.

12. Mackworth-Young CG, Loizou S, Walport MJ. Primary antiphospholipid syndrome: features of patients with raised anticardiolipin antibodies and no other disorder. Ann Rheum Dis 48: 362-7, 1989.

13. Freitas MV, Silva LM, Petean FC, et al. Síndrome do anticorpo antifosfolípide: estudo comparativo das formas primária e secundária. Rev Bras Reumatol 43: 153-9, 2003.

14. Gourley IS, McMillan SA, Bell AL. Clinical features associated with a positive anticardiolipin antibody in Irish patients with systemic lupus erythematosus. Clin Rheumatol 15: 457-60, 1996.

15. Mok MY, Chan EY, Fong DY, et al. Antiphospholipid antibody profiles and their clinical associations in Chinese patients with systemic lupus erythematosus. J Rheumatol 32: 622-8, 2005.

16. Wilson WA, Gharavi AE, Koike T, et al. International consensus statement on preliminary classification criteria for definite antiphospholipid syndrome: report of an international workshop. Arthritis Rheum 42: 1309-11, 1999.

17. Tan EM, Cohen AS, Fries JF, et al. The 1982 revised criteria for the classification of systemic lupus erythematosus. Arthritis Rheum 25: 1271-7, 1982.

18. Kurata N, Tan EM. Identification of antibody to nuclear acidic antigens by counterimmunoelectrophoresis. Arthritis Rheum 19: 574-80, 1976.

19. Elkon KB, Culhane L. Partial immunochemical characterization of the Ro and La proteins using antibodies from patients with the sicca syndrome and lupus erythematosus. J Immunol 132: 2350-6, 1984.

20. Gharavi AE, Harris EN, Asherson RA, Hughes GRV. Anticardiolipin antibodies: isotype distribution and phospholipid specificity. Ann Rheum Dis 46: 1-6, 1987. 